\title{
Low levels of linoleic acid and $\alpha$-linolenic acid and high levels of arachidonic acid in plasma phospholipids are associated with hypertension
}

\author{
IKUYO TSUKAMOTO $^{1,2}$ and SHIORI SUGAWARA ${ }^{1,3}$ \\ ${ }^{1}$ Department of Food Science and Nutrition, Nara Women's University, Kitauoya-Nishimachi, Nara 630-8506; \\ ${ }^{2}$ Faculty of Clinical Nutrition, Hiroshima International University, Hirokoshingai, Kure, Hiroshima 737-0112; \\ ${ }^{3}$ Department of Health and Nutrition, Sendai Shirayuri Women's College, \\ Honda-Cho, Izumi-ku, Sendai 981-3107, Japan
}

Received September 11, 2017; Accepted October 10, 2017

DOI: 10.3892/br.2017.1015

\begin{abstract}
Dietary fat is an important determinant in the development and progression of high blood pressure (BP), a major risk factor for cardiovascular diseases and mortality. The aim of the present study was to determine the association between plasma phospholipid fatty acids and hypertension in Japanese men. The plasma level of linoleic acid (LA) in the subjects with hypertension (systolic BP $\geq 140 \mathrm{mmHg}$ and/or diastolic BP $\geq 90 \mathrm{mmHg}$ ) was identified to be significantly higher than that in the healthy controls. Following adjustment for age, body mass index, physical activity, smoking status, alcohol consumption, salt intake, and serum levels of glucose and hemoglobin A1c, higher plasma levels of LA and $\alpha$-linolenic acid (ALA), and lower levels of arachidonic acid (AA) were significantly associated with a lower prevalence of hypertension. The odds ratio (OR) for the highest quartile (Q4) versus the lowest quartile $(\mathrm{Q} 1)$ of LA was $0.17(\mathrm{P}=0.003)$, the OR for Q4 versus Q1 of ALA was $0.26(\mathrm{P}=0.042)$ and the OR for Q4 versus Q1 of AA was $2.04(\mathrm{P}=0.047)$. These results indicate that elevated levels of LA and ALA, and reduced levels of AA in the plasma prevent hypertension.
\end{abstract}

Correspondence to: Professor Ikuyo Tsukamoto, Faculty of Clinical Nutrition, Hiroshima International University, 5-1-1 Hirokoshingai, Kure, Hiroshima 737-0112, Japan

E-mail: itsuka@cc.nara-wu.ac.jp; itsuka@ps.hirokoku-u.ac.jp

Abbreviations: AA, arachidonic acid; ALA, $\alpha$-linolenic acid; BMI, body mass index; BP, blood pressure; DHA, docosahexaenoic acid; EPA, eicosapentaenoic acid; LA, linoleic acid; MUFA, monounsaturated fatty acid; FA, fatty acid; OR, odds ratio; PL, phospholipid; PUFA, polyunsaturated fatty acid; SFA, saturated fatty acid

Key words: hypertension, plasma fatty acid, $\alpha$-linolenic acid, linoleic acid, arachidonic acid

\section{Introduction}

High blood pressure (BP) is a major risk factor for cardiovascular diseases and mortality. The progressive increase in BP over time is directly linked to vascular aging, characterized by endothelial dysfunction, arterial stiffening and inflammation (1).

Preventing the increase in BP is a major health problem globally, particularly in Japan with its increasing number of elderly people.

Dietary fat is an important determinant in the development and progression of high BP. Animal studies have demonstrated that diets high in saturated fatty acid (SFA) increase BP $(2,3)$, whereas diets enriched with n-3 polyunsaturated fatty acid (PUFA) protect against BP elevation (4-6). However, epidemiologic evidence regarding the association between various subtypes and individual FA intake, and the risk of developing hypertension remains limited and inconsistent (7-9). No significant correlations have been observed between hypertension and intakes of SFA and PUFA $(7,8)$. However, higher SFA and lower PUFA consumption have been associated with higher BP (9). A summary of the International Study of Macro/Micronutrients and Blood Pressure (INTERMAP) reported that total PUFA, linoleic acid (LA), total n-3 FA, and $\alpha$-linolenic acid (ALA), were inversely associated with BP (10). Data from meta-analyses $(11,12)$ and clinical trials $(13,14)$ have indicated that n-3 PUFA supplementation dose-dependently reduces $\mathrm{BP}$ in hypertensive patients, but not in normotensive individuals. A large-scale, population-based INTERMAP indicated that dietary intake of n-3 PUFA was inversely associated with BP levels in middle-aged normotensive individuals (15). A potential reason for the inconsistencies across studies may be attributable to the limited reliability of dietary measurement.

In contrast to dietary questionnaires, the FA composition of plasma cholesterol esters, phospholipids (PL), or the erythrocyte membrane is a reasonably accurate, objective measure that reflects dietary consumption and relevant biological processes (16-20). The serum/plasma levels of PUFA were significantly correlated with the intake of polyunsaturated 
fat (16), while its levels of 14:0, 16:0, 18:0, and monounsaturated FA may reflect a diet rich in saturated fat, due to their high correlations with saturated fat (19). Prior observational studies examined associations between serum/plasma or erythrocyte membrane FA and BP. However, their findings were controversial (21-26). Stearic acid, palmitoleic acid, n-9 eicosatrienoic acid, and dihomogammalinoleic acid in serum cholesterol esters (21), or total fatty acids, SFA, and LA in plasma PL were indicated to be associated with BP (22). Hypertensive men had lower C22:6/C20:5 [n-3 $\delta-6$ desaturase (D6D) index], PUFA and polyunsaturated/saturated fatty acid $(\mathrm{P} / \mathrm{S})$ ratios in their serum free FA composition (24). Reduced levels of LA and $\mathrm{P} / \mathrm{S}$ ratio, and elevated levels of palmitic acid and arachidonic acids (AA) in plasma cholesterol esters were associated with a higher risk of hypertension (23). A higher $\mathrm{P} / \mathrm{S}$ ratio in the erythrocyte membrane is associated with the risk of hypertension in middle-aged and older women (25). Erythrocyte long-chain (LC) n-3PUFA (3) or LA (26) was associated with reduced BP levels. Thus, there is need for further investigation to elucidate the differential effects of FA on BP.

In this study, to obtain a dietary recommendation for preventing hypertension, the association between plasma FA composition and estimated desaturase activity, and hypertension was investigated in Japanese men.

\section{Materials and methods}

Subjects. The study population comprised of 315 Japanese men (52.2 \pm 7.3 years old) who participated in a health examination conducted in 2007, 2008, 2009, 2011 and 2013 at the Nara Health Promotion Center (Nara, Japan). All participants who had received and were presently receiving medical treatment and medication for cancer, hypertension, diabetes, dyslipidemia or cardiovascular disease were excluded following a face-toface interview with a physician. The study was designed in accordance with the principles of the Declaration of Helsinki of the World Medical Association and approved by the Ethics Committee of Nara Women's University (Nara, Japan). At the time of enrollment, written informed consent was obtained from each participant.

Measurement. The questionnaires were administered to the participants. The participants underwent routine health examinations, anthropometric and BP measurements, and the collection of fasting blood samples. A face-to-face interview was conducted by trained interviewers using questionnaires about the medical condition, medication use, lifestyle of physical activity ( $>30 \mathrm{~min}$ of exercise or $1 \mathrm{~h}$ of walking per day), present smoking, and alcohol drinking ( $\geq 20 \mathrm{~g}$ ethanol per day) and a previously validated food frequency questionnaire (59).

Anthropometry. The body mass index (BMI) was calculated as the body weight (kg) divided by the square of the height (m). The waist circumference (WC) was measured using an anthropometric measuring tape at a horizontal plane midway between the lowest rib and the iliac crest. BP was measured in triplicate with a validated semi-automatic sphygmomanometer after a minimum of $5 \mathrm{~min}$ rest in a seated position, in a quiet room, with no physical activity in the preceding half hour.
Laboratory measurements. All serum and plasma samples were obtained in the fasted state. Routine clinical parameters, such as serum triacylglycerol, total cholesterol, low-density lipoprotein (LD)-cholesterol, high-density lipoprotein (HDL)-cholesterol, fasting blood glucose, fasting insulin, and hemoglobin $(\mathrm{Hb}) \mathrm{A} 1 \mathrm{c}$ were measured. The insulin resistance index was calculated according to the homeostasis model assessment (HOMA-IR) where HOMA-IR = fasting insulin $(\mu \mathrm{U} / \mathrm{ml}) \times$ fasting glucose $(\mathrm{mg} / \mathrm{dl}) / 405$.

FA analysis. Plasma was separated by centrifugation of fasting blood samples containing ethylenediaminetetraacetic acid-2Na for $15 \mathrm{~min}$ at $1,600 \mathrm{x} \mathrm{g}$ at $4^{\circ} \mathrm{C}$, and stored at $-80^{\circ} \mathrm{C}$. Total lipids were extracted from the plasma according to the method of Bligh and Dyer (27). PL were separated by thin-layer chromatography using silica gel plates (Silica Gel 60; Merck KGaA, Darmstadt, Germany) with a solvent system of petroleum ether to ethylether to acetic acid $(80: 20: 1 ; \mathrm{v} / \mathrm{v} / \mathrm{v})$. The spot corresponding to PL was scraped from the plate and transmethylated with $2 \mathrm{ml}$ acetyl chloride: methanol $(5: 50 ; \mathrm{v} / \mathrm{v})$ at $90^{\circ} \mathrm{C}$ for $2 \mathrm{~h}$. Heptadecanoic acid (17:0) served as an internal standard. FA methyl esters were analyzed by gas-liquid chromatography (GC-2014; Shimadzu Corp., Kyoto, Japan) with a $25 \mathrm{~m}$ x $0.5 \mathrm{~mm}$ capillary column (HR-SS-10; Shinwa Chemical Industries Ltd., Kyoto, Japan) as previously described (28).

Estimation of desaturase activity. The estimated desaturase activity was calculated by the ratio of the FAs in serum PL as follows: $\delta-9$ desaturase activity $(\mathrm{D} 9 \mathrm{D})=[16: 1 \mathrm{n}-7 / 16: 0], \delta-6$ desaturase $(D 6 D)=[18: 3 n-6 / 18: 2 n-6]$ and $\delta-5$ desaturase $(D 5 D)=[20: 4 n-6 / 20: 3 n-6]$.

Statistical analysis. Statistical analyses were performed using SPSS software version 23.0 (IBM, Inc., Armonk, NY, USA). All statistical tests were two-sided and $\mathrm{P}<0.05$ was considered to indicate a statistically significant difference. Continuous data were expressed as the mean \pm standard deviation (SD). The distribution of continuous variables was examined for normality by Shapiro-Wilk's test. Categorical data were expressed as a proportion (\%). Differences in the continuous and categorical variables between two groups was examined using an unpaired Student's t-test (normally distributed data) or the Mann-Whitney U test (non-normally distributed data) and by the Chi-square test, respectively.

Logistic regression analysis was performed to calculate the odds ratios (OR) and $95 \%$ confidence intervals (CIs) to examine the associations between the prevalence of hypertension across quartiles of plasma PL FA and estimated desaturase activities considering the lowest quartile as the reference and controlling for potential confounding factors (age, BMI, physical activity, smoking status, alcohol drinking, salt intake, and serum levels of glucose and $\mathrm{HbAlc}$ ).

\section{Results}

Characteristics of subjects. The subjects were 35 to 77 years old (those in their thirties, forties, fifties, sixties, and seventies were $12,106,155,39$, and 3 men, respectively). Of the total population, $61.0,7.9$ and $13.3 \%$ were overweight or obese (BMI $\geq 25$ ), had diabetes (fasting glucose $\geq 126 \mathrm{mg} / \mathrm{dl}$ ), 
Table I. Characteristics of group subjects.

\begin{tabular}{|c|c|c|c|}
\hline Variable & $\begin{array}{l}\text { Normal } \\
(n=246)\end{array}$ & $\begin{array}{l}\text { Hypertension } \\
\qquad(n=69)\end{array}$ & P-value \\
\hline Age (years) & $51.8 \pm 7.1$ & $53.5 \pm 7.9$ & 0.097 \\
\hline \multicolumn{4}{|l|}{ Lifestyle factors } \\
\hline Physical activity (\% yes) & 35.3 & 49.1 & 0.067 \\
\hline Smoking (\% yes) & 28.4 & 17.5 & 0.107 \\
\hline Alcohol consumption ( $\geq 20 \mathrm{~g} / \mathrm{day}, \%$ yes) & 44.7 & 52.6 & 0.256 \\
\hline Salt intake (g/day) & $8.7 \pm 2.9$ & $8.4 \pm 3.0$ & 0.255 \\
\hline \multicolumn{4}{|l|}{ Anthropometry indicators } \\
\hline Body mass index $\left(\mathrm{kg} / \mathrm{m}^{2}\right)$ & $25.4 \pm 3.1$ & $25.9 \pm 2.7$ & 0.160 \\
\hline Waist circumference $(\mathrm{cm})$ & $90.1 \pm 8.0$ & $91.3 \pm 6.6$ & 0.297 \\
\hline Systolic BP (mmHg) & $124 \pm 10$ & $152 \pm 10$ & $<0.001$ \\
\hline Diastolic BP (mmHg) & $79 \pm 8$ & $96 \pm 10$ & $<0.001$ \\
\hline \multicolumn{4}{|l|}{ Biochemical parameters } \\
\hline Triacylglycerol (mg/dl) & $141.0 \pm 92.3$ & $150.9 \pm 84.1$ & 0.475 \\
\hline Total-cholesterol (mg/dl) & $206.5 \pm 31.0$ & $212.1 \pm 29.5$ & 0.182 \\
\hline HDL-cholesterol (mg/dl) & $53.1 \pm 13.7$ & $54.7 \pm 13.1$ & 0.249 \\
\hline LDL-cholesterol (mg/dl) & $127.6 \pm 29.3$ & $128.9 \pm 28.6$ & 0.429 \\
\hline Glucose (mg/dl) & $102 \pm 14$ & $108 \pm 18$ & 0.001 \\
\hline Hemoglobin A1c (\%) & $5.2 \pm 0.5$ & $5.5 \pm 0.5$ & 0.022 \\
\hline Insulin $(\mu \mathrm{U} / \mathrm{ml})$ & $8.8 \pm 5.8$ & $9.5 \pm 7.2$ & 0.662 \\
\hline HOMA-IR & $2.25 \pm 1.67$ & $2.54 \pm 2.09$ & 0.374 \\
\hline
\end{tabular}

Data are expressed as the mean \pm standard deviation or percentage. BP, blood pressure; HDL, high-density lipoprotein; LDL, low-density lipoprotein; HOMA-IR, homeostasis model assessment-estimated insulin resistance.

or were elderly (age $\geq 60$ ), respectively. The subjects were divided into two groups as follows: Hypertension (systolic BP; $\mathrm{SBP} \geq 140 \mathrm{mmHg}$ and/or diastolic BP; DBP $\geq 90$ ) and normal. The characteristics of the two groups, normal $(n=246)$ and hypertension $(n=69)$ are presented in Table I. As expected, the SBP and DBP of the hypertension group were significantly higher than those of the normal group. However, age, BMI, WC, and lifestyle, such as physical activity, present smoking, alcohol consumption and salt intake were not significantly different between the two groups.

In addition, the clinical data of the subjects are presented in Table I. The indicators of lipid metabolism, the values of triacylglycerol, total cholesterol, HDL-cholesterol and LDL-cholesterol, were not significantly different between the two groups. The values of fasting glucose and HbAlc in the hypertension group were significantly higher than those in the normal group, but their values were standard levels. The HOMA-IR values of the two groups were similar.

FA composition of plasma PL. The FA composition of plasma $\mathrm{PL}$ in the two groups is presented in Table II. The level of LA was significantly lower in the hypertension than in the normal group. Except for LA, the levels of other FAs and the estimated desaturase activities did not differ significantly between the two groups.

Associations between hypertension and plasma PL FA. To determine the associations between the prevalence of hypertension across quartiles of plasma PL FA and estimated desaturase activities, considering the lowest quartile as the reference, logistic regression analysis was performed. The OR associated with hypertension by quartiles of plasma FA proportions and estimated desaturase activities are presented in Table III. In a crude regression model, a higher quartile of LA was associated with a decreased prevalence of hypertension. The analysis indicated that the increase in the mean LA levels (from 15 to $21 \mathrm{~mol} \%$ ) decreased the prevalence of hypertension to $39 \%$. Following adjustment for age, BMI, lifestyle, and serum levels of fasting glucose and HbAlc, higher quartiles of LA and ALA acid were significantly associated with a lower prevalence of hypertension, while the higher quartile of AA was associated with a higher prevalence of hypertension. The adjusted model demonstrated that the 1.4-times increase in the mean LA level (from 15 to $21 \mathrm{~mol} \%$ ) or 3.3-times increase in the mean ALA level (from 0.13 to 0.43 mol\%) decreased the prevalence of hypertension to 17 or $26 \%$, respectively. Conversely, the 1.5 times elevation of the mean AA level (from 5.68 to $8.47 \mathrm{~mol} \%$ ) increased the prevalence of hypertension to 2 -fold.

\section{Discussion}

The present study demonstrated that the plasma level of LA in the subjects with hypertension was significantly higher than that in those without hypertension. Furthermore, logistic regression analysis demonstrated that higher quartiles of LA were 
Table II. FA composition in plasma phospholipids between normal and hypertensive subjects.

\begin{tabular}{|c|c|c|c|}
\hline \multirow[b]{2}{*}{$\mathrm{FA}(\mathrm{mol} \%)$} & \multicolumn{3}{|c|}{ Groups } \\
\hline & Normal & Hypertension & P-value \\
\hline Myristic acid (14:0) & $0.68 \pm 0.37$ & $0.76 \pm 0.67$ & 0.490 \\
\hline Palmitic acid (16:0) & $32.43 \pm 3.85$ & $32.50 \pm 3.45$ & 0.976 \\
\hline Palmitoleic acid (16:1n-7) & $0.64 \pm 0.40$ & $0.71 \pm 0.35$ & 0.076 \\
\hline Stearic acid (18:0) & $13.61 \pm 1.49$ & $13.68 \pm 1.24$ & 0.727 \\
\hline Oleic acid (18:1n-9) & $9.65 \pm 1.68$ & $9.98 \pm 1.70$ & 0.159 \\
\hline Vaccenic acid (18:1n-7) & $1.68 \pm 0.66$ & $1.68 \pm 0.52$ & 0.589 \\
\hline Linoleic acid (18:2n-6) & $18.51 \pm 3.18$ & $17.34 \pm 2.67$ & 0.004 \\
\hline$\gamma$-Linolenic acid (18:3n-6) & $0.15 \pm 0.15$ & $0.16 \pm 0.16$ & 0.612 \\
\hline$\alpha$-Linolenic acid (18:3n-3) & $0.27 \pm 0.15$ & $0.26 \pm 0.17$ & 0.295 \\
\hline Arachidic acid (20:0) & $0.46 \pm 0.24$ & $0.50 \pm 0.28$ & 0.402 \\
\hline Eicosadienoic acid (20:2n-6) & $0.34 \pm 0.19$ & $0.35 \pm 0.20$ & 0.992 \\
\hline Dihomo- $\gamma$-linolenic acid $(20: 3 n-6)$ & $1.92 \pm 0.53$ & $1.96 \pm 0.47$ & 0.619 \\
\hline Arachidonic acid (20:4n-6) & $6.99 \pm 1.20$ & $7.29 \pm 1.37$ & 0.072 \\
\hline Behenic acid (22:0) & $0.84 \pm 0.50$ & $0.87 \pm 0.46$ & 0.690 \\
\hline Eicosapentaenoic acid (20:5n-3) & $2.11 \pm 1.10$ & $2.28 \pm 1.17$ & 0.304 \\
\hline Docosadienoic acid (22:2n-6) & $0.29 \pm 0.27$ & $0.26 \pm 0.23$ & 0.500 \\
\hline Lignoceric acid (24:0) & $0.76 \pm 0.43$ & $0.68 \pm 0.35$ & 0.165 \\
\hline Docosapentaenoic acid (22:5n-3) & $0.91 \pm 0.59$ & $1.01 \pm 0.75$ & 0.261 \\
\hline Docosahexaenoic acid (22:6n-3) & $4.82 \pm 1.28$ & $4.99 \pm 1.32$ & 0.343 \\
\hline Total SFA & $49.31 \pm 3.85$ & $49.57 \pm 3.40$ & 0.961 \\
\hline Total MUFA & $13.98 \pm 1.76$ & $14.16 \pm 1.78$ & 0.574 \\
\hline Total PUFA & $36.67 \pm 3.51$ & $36.23 \pm 3.18$ & 0.541 \\
\hline PUFA/SFA & $0.76 \pm 0.28$ & $0.74 \pm 0.11$ & 0.719 \\
\hline Total n-6 PUFA & $28.37 \pm 3.37$ & $27.51 \pm 2.87$ & 0.067 \\
\hline Total n-3 PUFA & $8.30 \pm 2.48$ & $8.72 \pm 2.77$ & 0.314 \\
\hline$n-6 / n-3$ & $3.80 \pm 1.47$ & $3.54 \pm 1.37$ & 0.163 \\
\hline D9D & $0.021 \pm 0.020$ & $0.023 \pm 0.012$ & 0.100 \\
\hline D6D & $0.008 \pm 0.009$ & $0.010 \pm 0.011$ & 0.354 \\
\hline D5D & $3.89 \pm 1.18$ & $3.92 \pm 1.12$ & 0.666 \\
\hline
\end{tabular}

Data are expressed as means \pm standard deviation. FA, fatty acid; SFA, saturated fatty acid; MUFA, monounsaturated fatty acid; PUFA, polyunsaturated fatty acid; D9D, $\delta-9$ desaturase; D6D, $\delta-6$ desaturase; D5D, $\delta-5$ desaturase.

associated with a lower prevalence of hypertension. The 1.4 fold increase in the mean levels of LA decreased the prevalence of hypertension to 0.39 and 0.17 fold in a crude model, and in the adjusted for age, BMI, lifestyle associated factors, and serum levels of fasting glucose and HbA1c, respectively. Previous studies have identified dietary (10) and plasma/erythrocyte membrane levels of LA $(22,23,26)$ to be inversely associated with BP, although the results are inconsistent $(3,24,25)$. The possibility of detecting an association between LA and BP may be limited by imprecise measures of dietary LA, the sample size, and the degree of statistical control for potential confounders. However, consistent with the present results, animal studies indicate that LA may reduce the BP by serving as a substrate for vasoactive prostaglandins (29) and promote the relaxation of vascular smooth muscle cells (30).

After adjusting for the confounding factors, logistic analysis indicated that higher quartiles of ALA, the essential n-3 PUFA, were associated with a lower prevalence of hypertension. Consistently, the mean BP was reported to be inversely associated with the dietary intake of ALA in a cross-sectional Finnish study (31), and the concentration of adipose tissue linolenic acid (32). However, ALA supplementation led to no significant difference in BP changes between participants taking supplementation compared with the placebo control groups (33). ALA may be converted to eicosapentaenoic acid [EPA; 20:5n-3], but only in a small percentage and the conversion of EPA to docosahexaenoic acid [DHA; 22:6n-3], if any, is particularly limited in mammals (34). These findings indicate that the effect of ALA would not be mediated by EPA and DHA. It has been reported that ALA, as well as oleic acid and LA affects the structure of the cell membrane and regulates cell function (35). The effects of ALA on the cell membrane, at least partially, may explain its association with BP $(35,36)$. 
Table III. Multivariate adjusted ORs and 95\% CI for hypertension by quartile of plasma phospholipid fatty acid composition.

\begin{tabular}{|c|c|c|c|c|c|}
\hline \multirow[b]{2}{*}{ Variable } & \multicolumn{5}{|c|}{ Quartiles of plasma phospholipid fatty acid composition } \\
\hline & Q1 (Lowest) & Q2 & Q3 & Q4 (Highest) & P-value \\
\hline \multicolumn{6}{|l|}{ Palmitic acid (16:0) } \\
\hline Median (mol \%) & 29.15 & 31.09 & 33.36 & 36.88 & \\
\hline Subjects with/without Hyp (n) & $18 / 61$ & $15 / 64$ & $19 / 60$ & $17 / 62$ & \\
\hline Unadjusted OR (95\% CI) & 1 (Reference) & $0.79(0.37-1.72)$ & $1.07(0.51-2.24)$ & $0.94(0.45-2.00)$ & 0.924 \\
\hline Adjusted OR $(95 \% \mathrm{CI})^{\mathrm{a}}$ & 1 (Reference) & $0.78(0.26-2.38)$ & $1.07(0.32-3.57)$ & $0.85(0.31-2.31)$ & 0.987 \\
\hline \multicolumn{6}{|l|}{ Stearic acid (18:0) } \\
\hline Median (mol \%) & 12.29 & 13.29 & 13.91 & 15.10 & \\
\hline Subjects with/without Hyp (n) & $16 / 63$ & $18 / 61$ & $17 / 62$ & $18 / 60$ & \\
\hline Unadjusted OR (95\% CI) & 1 (Reference) & $1.16(0.54-2.48)$ & $1.08(0.50-2.33)$ & $1.18(0.65-2.53)$ & 0.711 \\
\hline Adjusted OR $(95 \% \mathrm{CI})^{\mathrm{a}}$ & 1 (Reference) & $1.93(0.67-5.51)$ & $2.62(0.90-7.65)$ & $1.51(0.46-4.98)$ & 0.403 \\
\hline \multicolumn{6}{|l|}{ Palmitoleic acid (16:1n-7) } \\
\hline Median (mol \%) & 0.28 & 0.52 & 0.71 & 0.97 & \\
\hline Subjects with/without Hyp (n) & $17 / 62$ & $10 / 69$ & $20 / 59$ & $22 / 56$ & \\
\hline Unadjusted OR (95\% CI) & 1 (Reference) & $0.53(0.23-1.24)$ & $1.24(0.59-2.59)$ & $1.43(0.69-2.97)$ & 0.133 \\
\hline Adjusted OR $(95 \% \mathrm{CI})^{\mathrm{a}}$ & 1 (Reference) & $0.40(0.12-1.38)$ & $1.44(0.53-3.87)$ & $1.70(0.65-4.48)$ & 0.163 \\
\hline \multicolumn{6}{|l|}{ Oleic acid (18:1n-9) } \\
\hline Median (mol \%) & 8.01 & 9.06 & 10.01 & 11.61 & \\
\hline Subjects with/without Hyp (n) & $13 / 66$ & $17 / 62$ & $22 / 57$ & $17 / 61$ & \\
\hline Unadjusted OR (95\% CI) & 1 (Reference) & $1.39(0.63-3.10)$ & $1.96(0.91-4.24)$ & $1.42(0.64-3.15)$ & 0.367 \\
\hline Adjusted OR $(95 \% \mathrm{CI})^{\mathrm{a}}$ & 1 (Reference) & $0.63(0.23-1.71)$ & $1.20(0.42-3.44)$ & $0.81(0.28-2.41)$ & 0.954 \\
\hline \multicolumn{6}{|l|}{ Linoleic acid (18:2n-6) } \\
\hline Median (mol \%) & 15.04 & 17.43 & 18.97 & 21.01 & \\
\hline Subjects with/without Hyp (n) & $25 / 54$ & $20 / 59$ & $12 / 67$ & $12 / 66$ & \\
\hline Unadjusted OR (95\% CI) & 1 (Reference) & $0.73(0.37-1.47)$ & $0.39(0.18-0.84)$ & $0.39(0.18-0.85)$ & 0.006 \\
\hline Adjusted OR $(95 \% \mathrm{CI})^{\mathrm{a}}$ & 1 (Reference) & $0.55(0.20-1.49)$ & $0.35(0.13-0.96)$ & $0.17(0.05-0.61)$ & 0.003 \\
\hline \multicolumn{6}{|l|}{$\gamma$-linolenic acid $(18: 3 n-6)$} \\
\hline Median (mol \%) & 0.04 & 0.08 & 0.13 & 0.31 & \\
\hline Subjects with/without Hyp (n) & $16 / 63$ & $16 / 63$ & $22 / 57$ & $15 / 63$ & \\
\hline Unadjusted OR (95\% CI) & 1 (Reference) & $1.00(0.46-2.17)$ & $1.52(0.73-3.18)$ & $0.94(0.43-2.06)$ & 0.799 \\
\hline Adjusted OR $(95 \% \mathrm{CI})^{\mathrm{a}}$ & 1 (Reference) & $1.57(0.53-4.62)$ & $2.56(0.83-7.84)$ & $2.04(0.65-6.39)$ & 0.310 \\
\hline \multicolumn{6}{|l|}{$\begin{array}{l}\text { Dihomo- } \gamma \text {-linolenic } \\
\text { acid }(20: 3 n-6)\end{array}$} \\
\hline Median (mol\%) & 1.34 & 1.74 & 2.05 & 2.56 & \\
\hline Subjects with/without Hyp (n) & $15 / 64$ & $18 / 61$ & $20 / 59$ & $16 / 62$ & \\
\hline Unadjusted OR (95\% CI) & 1 (Reference) & $1.26(0.58-2.72)$ & $1.44(0.68-3.08)$ & $1.10(0.50-2.42)$ & 0.791 \\
\hline Adjusted OR $(95 \% \mathrm{CI})^{\mathrm{a}}$ & 1 (Reference) & $1.29(0.45-3.75)$ & $1.65(0.56-4.83)$ & $1.01(0.30-3.42)$ & 0.915 \\
\hline \multicolumn{6}{|l|}{ AA $(20: 4 n-6)$} \\
\hline Median (mol \%) & 5.68 & 6.61 & 7.50 & 8.47 & \\
\hline Subjects with/without Hyp (n) & $15 / 64$ & $8 / 71$ & $28 / 51$ & $18 / 60$ & \\
\hline Unadjusted OR (95\% CI) & 1 (Reference) & $0.48(019-1.21)$ & $2.34(1.13-4.85)$ & $1.28(0.59-2.77)$ & 0.080 \\
\hline Adjusted OR $(95 \% \mathrm{CI})^{\mathrm{a}}$ & 1 (Reference) & $0.38(0.10-1.44)$ & $2.07(0.72-5.96)$ & $2.04(0.71-5.85)$ & 0.047 \\
\hline \multicolumn{6}{|l|}{$\operatorname{ALA}(18: 3 n-3)$} \\
\hline Median (mol \%) & 0.13 & 0.2 & 0.28 & 0.43 & \\
\hline Subjects with/without Hyp (n) & $22 / 57$ & $16 / 63$ & $18 / 61$ & $13 / 65$ & \\
\hline Unadjusted OR (95\% CI) & 1 (Reference) & $0.66(0.32-1.38)$ & $0.77(0.37-1.57)$ & $0.52(0.24-1.12)$ & 0.139 \\
\hline Adjusted OR $(95 \% \text { CI })^{\mathrm{a}}$ & 1 (Reference) & $0.55(0.21-1.43)$ & $0.61(0.21-1.78)$ & $0.26(0.07-0.95)$ & 0.042 \\
\hline \multicolumn{6}{|l|}{$\operatorname{EPA}(20: 5 n-3)$} \\
\hline Median (mol\%) & 0.99 & 1.64 & 2.27 & 3.40 & \\
\hline Subjects with/without Hyp (n) & $16 / 63$ & $16 / 63$ & $15 / 64$ & $22 / 56$ & \\
\hline
\end{tabular}


Table III. Continued.

Quartiles of plasma phospholipid fatty acid composition

\begin{tabular}{|c|c|c|c|c|c|}
\hline Variable & Q1 (Lowest) & Q2 & Q3 & Q4 (Highest) & P-value \\
\hline \multicolumn{6}{|l|}{ EPA $(20: 5 n-3)$} \\
\hline Unadjusted OR (95\% CI) & 1 (Reference) & $1.00(0.46-2.17)$ & $0.92(0.42-2.03)$ & $1.55(0.74-3.24)$ & 0.218 \\
\hline Adjusted OR $(95 \% \mathrm{CI})^{\mathrm{a}}$ & 1 (Reference) & $2.00(0.70-5.70)$ & $1.30(0.41-4.11)$ & $1.38(0.43-4.38)$ & 0.879 \\
\hline \multicolumn{6}{|l|}{ DHA (22:6n-3) } \\
\hline Median (mol\%) & 3.43 & 4.41 & 5.33 & 6.30 & \\
\hline Subjects with/without Hyp (n) & $15 / 65$ & $21 / 58$ & $13 / 66$ & $21 / 57$ & \\
\hline Unadjusted OR (95\% CI) & 1 (Reference) & $1.68(0.78-3.61)$ & $0.92(0.40-2.10)$ & $1.71(0.80-3.67)$ & 0.393 \\
\hline Adjusted OR $(95 \% \mathrm{CI})^{\mathrm{a}}$ & 1 (Reference) & $2.26(0.78-6.54)$ & $0.85(0.25-2.95)$ & $1.51(0.52-4.44)$ & 0.802 \\
\hline \multicolumn{6}{|l|}{ n-3 PUFA (ALA+EPA+DHA) } \\
\hline Median (mol\%) & 5.51 & 7.2 & 8.97 & 11.52 & \\
\hline Subjects with/without Hyp (n) & $17 / 62$ & $16 / 63$ & $13 / 66$ & $23 / 55$ & \\
\hline Unadjusted OR (95\% CI) & 1 (Reference) & $0.93(0.43-2.00)$ & $0.72(0.32-1.60)$ & $1.53(0.74-3.15)$ & 0.259 \\
\hline Adjusted OR $(95 \% \mathrm{CI})^{\mathrm{a}}$ & 1 (Reference) & $1.43(0.53-3.88)$ & $0.67(0.20-2.26)$ & $1.21(0.44-3.29)$ & 0.960 \\
\hline \multicolumn{6}{|l|}{ Ratio of $n-6$ to n- 3 PUFA } \\
\hline Median & 2.31 & 3.09 & 3.97 & 5.17 & \\
\hline Subjects with/without Hyp (n) & $23 / 56$ & $13 / 66$ & $18 / 61$ & $15 / 63$ & \\
\hline Unadjusted OR (95\% CI) & 1 (Reference) & $0.48(0.22-1.03)$ & $0.72(0.35-1.47)$ & $0.58(0.28-1.22)$ & 0.295 \\
\hline Adjusted OR $(95 \% \mathrm{CI})^{\mathrm{a}}$ & 1 (Reference) & $0.82(0.25-2.64)$ & $1.14(0.40-3.25)$ & $1.03(0.36-2.99$ & 0.843 \\
\hline \multicolumn{6}{|l|}{ D9D } \\
\hline Median & 0.0079 & 0.0163 & 0.0222 & 0.0326 & \\
\hline Subjects with/without Hyp (n) & $16 / 63$ & $13 / 66$ & $19 / 60$ & $21 / 57$ & \\
\hline Unadjusted OR (95\% CI) & 1 (Reference) & $0.78(0.35-1.74)$ & $1.25(0.59-2.65)$ & $1.45(0.69-3.05)$ & 0.199 \\
\hline Adjusted OR $(95 \% \mathrm{CI})^{\mathrm{a}}$ & 1 (Reference) & $0.55(0.17-1.73)$ & $1.36(0.46-4.02)$ & $1.78(0.71-4.44)$ & 0.141 \\
\hline \multicolumn{6}{|l|}{ D6D } \\
\hline Median & 0.002 & 0.0047 & 0.0075 & 0.0175 & \\
\hline Subjects with/without Hyp (n) & $15 / 64$ & $15 / 64$ & $21 / 58$ & $18 / 60$ & \\
\hline Unadjusted OR (95\% CI) & 1 (Reference) & $1.00(0.45-2.22)$ & $1.55(0.73-3.28)$ & $1.28(0.59-2.77)$ & 0.510 \\
\hline Adjusted OR $(95 \% \mathrm{CI})^{\mathrm{a}}$ & 1 (Reference) & $1.35(0.43-4.23)$ & $2.08(0.71-6.07)$ & $2.04(0.67-6.18)$ & 0.226 \\
\hline \multicolumn{6}{|l|}{ D5D } \\
\hline Median & 2.74 & 3.40 & 4.07 & 5.22 & \\
\hline Subjects with/without Hyp (n) & $16 / 63$ & $17 / 62$ & $16 / 63$ & $20 / 58$ & \\
\hline Unadjusted OR (95\% CI) & 1 (Reference) & $1.08(0.50-2.33)$ & $1.00(0.46-2.17)$ & $1.36(0.64-2.87)$ & 0.431 \\
\hline Adjusted OR $(95 \% \mathrm{CI})^{\mathrm{a}}$ & 1 (Reference) & $1.38(0.42-4.53)$ & $0.98(0.27-3.62)$ & $2.13(0.63-7.23)$ & 0.198 \\
\hline
\end{tabular}

${ }^{\text {a} A d j u s t e d ~ f o r ~ a g e, ~ B M I, ~ p h y s i c a l ~ a c t i v i t y ~(y e s ~ o r ~ n o), ~ c u r r e n t ~ s m o k i n g ~(y e s ~ o r ~ n o), ~ a l c o h o l ~ c o n s u m p t i o n ~(~} \geq 20 \mathrm{~g} /$ day or no), salt intake and serum levels of glucose and hemoglobin A1c. Hyp, hypertension; AA, arachidonic acid; ALA, $\alpha$-linolenic acid; EPA, eicosapentaenoic acid; DHA, docosahexaenoic acid; BMI, body mass index; OR, odds ratio; CI, confidence interval; D9D, $\delta-9$ desaturase; D6D, $\delta-6$ desaturase; D5D, $\delta$-5 desaturase.

The long-chain n-3 fatty acids, EPA and DHA are considered to have an antihypertensive effect attributed to anti-inflammatory n-3 PUFA metabolites in eicosanoid/prostaglandin metabolism. EPA and DHA share the same metabolic pathways of AA and their competition with AA may decrease thromboxane $\mathrm{A}_{2}$ production, thus shifting the balance between thromboxane $\mathrm{A}_{2}$ and prostacyclin $\mathrm{I}_{2}$ toward a more favorable vasodilatory condition. Epidemiological and clinical data demonstrate that a high dietary intake of fish oil (EPA and DHA) may have beneficial effects on cardiovascular health and be associated with lower SBP and DBP $(37,38)$.

However, in the present study, neither EPA nor DHA was significantly associated with hypertension. This was also in contrast to some reports from clinical trials that dietary supplementation with fish oil (predominantly EPA and DHA) reduced BP in individuals with untreated hypertension, as well as numerous clinical and experimental studies (23). Recently, however, a review reported that current evidence supported the hypothesis that dietary supplementation of n-3 PUFA 
decreased the BP levels significantly only in hypertensive patients who showed an increase in the cell membrane content of these FAs, and this effect of n-3 PUFA on the BP was rather mild and not dose-associated (39).

A higher level of AA was associated with an increase in the prevalence of hypertension after adjusting for the confounding factors during logistic analysis in the present study. The Atherosclerosis Risk In Communities study indicated that a lower plasma LA level and higher plasma levels of palmitic acid and AA were associated with a higher risk of hypertension (23). AA may act through changes in eicosanoid/prostaglandin metabolism and the balance between thromboxane $\mathrm{A}_{2}$ (a vasoconstrictor agent) and prostacyclin (a vasodilating agent).

There were a number of limitations in the present study. As the participants were limited to Japanese men, our findings may not be generalizable to other populations. Due to the relatively small sample size, it is possible that associations between other FAs and BP may have been detectable in a larger study. Although the confounding variables were controlled, the possibility that unknown or unmeasured confounders account for the observed associations cannot be ruled out. Finally, the cross-sectional design of the present study mandates that inferences regarding causality be made with caution.

In conclusion, two essential FAs (LA and ALA) and AA were associated with hypertension. Higher plasma levels of LA and ALA, and lower levels of AA were associated with the reduced prevalence of hypertension. The plasma levels of these FAs reflect the dietary intakes (16-19), indicating that higher intakes of LA and ALA, and lower intakes of AA, prevent an increase in BP. Thus, dietary intake of FAs may be an important determinant in the prevention of hypertension.

\section{Acknowledgements}

The authors would like to thank the clinical support of Dr Takemi Akahane and Dr Kennichi Fukui of the Nara Health Promotion Center.

\section{References}

1. Sun Z: Aging, arterial stiffness, and hypertension. Hypertension 65: 252-256, 2015.

2. Gerber RT, Holemans K, O'Brien-Coker I, Mallet AI, van Bree R, Van Assche FA and Poston L: Cholesterol-independent endothelial dysfunction in virgin and pregnant rats fed a diet high in saturated fat. J Physiol 517: 607-616, 1999.

3. Zeng FF, Sun LL, Liu YH, Xu Y, Guan K, Ling WH and Chen YM: Higher erythrocyte n-3 PUFAs are associated with decreased blood pressure in middle-aged and elderly Chinese adults. J Nutr 144: 1240-1246, 2014

4. Rousseau D, Moreau D, Raederstorff D, Sergiel JP, Rupp H, Muggli R and Grynberg A: Is a dietary n-3 fatty acid supplement able to influence the cardiac effect of the psychological stress? Mol Cell Biochem 178: 353-366, 1998.

5. Rousseau D, Héliès-Toussaint C, Raederstorff D, Moreau D and Grynberg A: Dietary n-3 polyunsaturated fatty acids affect the development of renovascular hypertension in rats. Mol Cell Biochem 225: 109-119, 2001.

6. Rousseau D, Héliès-Toussaint C, Moreau D, Raederstorff D and Grynberg A: Dietary n-3 PUFAs affect the blood pressure rise and cardiac impairments in a hyperinsulinemia rat model in vivo. Am J Physiol Heart Circ Physiol 285: H1294-H1302, 2003.
7. Witteman JC, Willett WC, Stampfer MJ, Colditz GA, Sacks FM, Speizer FE, Rosner B and Hennekens CH: A prospective study of nutritional factors and hypertension among US women. Circulation 80: 1320-1327, 1989.

8. Ascherio A, Rimm EB, Giovannucci EL, Colditz GA, Rosner B, Willett WC, Sacks F and Stampfer MJ: A prospective study of nutritional factors and hypertension among US men. Circulation 86: 1475-1484, 1992.

9. Livingstone KM, Givens DI, Cockcroft JR, Pickering JE and Lovegrove JA: Is fatty acid intake a predictor of arterial stiffness and blood pressure in men? Evidence from the Caerphilly Prospective Study. Nutr Metab Cardiovasc Dis 23: 1079-1085, 2013.

10. Chan Q, Stamler J, Griep LM, Daviglus ML, Horn LV and Elliott P: An update on nutrients and blood pressure. J Atheroscler Thromb 23: 276-289, 2016.

11. Miller PE, Van Elswyk M and Alexander DD: Long-chain omega-3 fatty acids eicosapentaenoic acid and docosahexaenoic acid and blood pressure: A meta-analysis of randomized controlled trials. Am J Hypertens 27: 885-896, 2014.

12. Morris MC, Sacks F and Rosner B: Does fish oil lower blood pressure? A meta-analysis of controlled trials. Circulation 88: 523-533, 1993.

13. Root M, Collier SR, Zwetsloot KA, West KL and McGinn MC: A randomized trial of fish oil omega-3 fatty acids on arterial health, inflammation, and metabolic syndrome in a young healthy population. Nutr J 12: 40, 2013.

14. Wang S, Ma AQ, Song SW, Quan QH, Zhao XF and Zheng XH: Fish oil supplementation improves large arterial elasticity in overweight hypertensive patients. Eur J Clin Nutr 62: 1426-1431, 2008.

15. Ueshima H, Stamler J, Elliott P, Chan Q, Brown IJ, Carnethon MR, Daviglus ML, He K, Moag-Stahlberg A, Rodriguez BL, et al: INTERMAP research group: Food omega-3 fatty acid intake of individuals (total, linolenic acid, long-chain) and their blood pressure: INTERMAP study. Hypertension 50: 313-319, 2007.

16. Nikkari T, Luukkainen P, Pietinen P and Puska P: Fatty acid composition of serum lipid fractions in relation to gender and quality of dietary fat. Ann Med 27: 491-498, 1995.

17. Katan MB, Deslypere JP, van Birgelen AP, Penders M and Zegwaard M: Kinetics of the incorporation of dietary fatty acids into serum cholesteryl esters, erythrocyte membranes, and adipose tissue: An 18-month controlled study. J Lipid Res 38: 2012-2022, 1997.

18. Dougherty RM, Galli C, Ferro-Luzzi A and Iacono JM: Lipid and phospholipid fatty acid composition of plasma, red blood cells, and platelets and how they are affected by dietary lipids: A study of normal subjects from Italy, Finland, and the USA. Am J Clin Nutr 45: 443-455, 1987.

19. Ma J, Folsom AR, Shahar E and Eckfeldt JH: The atherosclerosis risk in communities (ARIC) study investigators: Plasma fatty acid composition as an indicator of habitual dietary fat intake in middle-aged adults. Am J Clin Nutr 62: 564-571, 1995.

20. Vessby B, Gustafsson IB, Tengblad S, Boberg M and Andersson A: Desaturation and elongation of fatty acids and insulin action. Ann N Y Acad Sci 967: 183-195, 2002.

21. Simon JA, Fong J and Bernert JT Jr: Serum fatty acids and blood pressure. Hypertension 27: 303-307, 1996.

22. Grimsgaard S, Bonaa KH, Jacobsen BK and Bjerve KS: Plasma saturated and linoleic fatty acids are independently associated with blood pressure. Hypertension 34: 478-483, 1999.

23. Zheng ZJ, Folsom AR, Ma J, Arnett DK, McGovern PG and Eckfeldt JH: Plasma fatty acid composition and 6-year incidence of hypertension in middle-aged adults: The atherosclerosis risk in communities (ARIC) study. Am J Epidemiol 150: 492-500, 1999.

24. Wang S, Ma A, Song S, Quan Q, Zhao X and Zheng X: Fasting serum free fatty acid composition, waist/hip ratio and insulin activity in essential hypertensive patients. Hypertens Res 31: 623-632, 2008.

25. Wang L, Tsai M, Manson JE, Djousse L, Gaziano JM, Buring JE and Sesso HD: Erythrocyte fatty acid composition is associated with the risk of hypertension in middle-aged and older women. J Nutr 141: 1691-1697, 2011

26. Yang B, Ding F, Yan J, Ye XW, Xu XL, Wang FL, Li D and Yu W: Exploratory serum fatty acid patterns associated with blood pressure in community-dwelling middle-aged and elderly Chinese. Lipids Health Dis 15: 58, 2016.

27. Bligh EG and Dyer WJ: A rapid method of total lipid extraction and purification. Can J Biochem Physiol 37: 911-917, 1959. 
28. Kawashima A, Sugawara S, Okita M, Akahane T, Fukui K, Hashiuchi M, Kataoka C and Tsukamoto I: Plasma fatty acid composition, estimated desaturase activities, and intakes of energy and nutrient in Japanese men with abdominal obesity or metabolic syndrome. J Nutr Sci Vitaminol 55: 400-406, 2009.

29. Iacono JM and Dougherty RM: Effects of polyunsaturated fats on blood pressure. Annu Rev Nutr 13: 243-260, 1993.

30. Pomposiello SI, Alva M, Wilde DW and Carretero OA: Linoleic acid induces relaxation and hyperpolarization of the pig coronary artery. Hypertension 31: 615-620, 1998.

31. Salonen JT: Dietary fats, antioxidants and blood pressure. Ann Med 23: 295-298, 1991.

32. Berry EM and Hirsch J: Does dietary linolenic acid influence blood pressure? Am J Clin Nutr 44: 336-340, 1986.

33. Wendland E, Farmer A, Glasziou P and Neil A: Effect of alpha linolenic acid on cardiovascular risk markers: A systematic review. Heart 92: 166-169, 2006.

34. Harris WS, Miller M, Tighe AP, Davidson MH and Schaefer EJ Omega-3 fatty acids and coronary heart disease risk: Clinical and mechanistic perspectives. Atherosclerosis 197: 12-24, 2008.

35. Ibarguren M, López DJ and Escribá PV: The effect of natural and synthetic fatty acids on membrane structure, microdomain organization, cellular functions and human health. Biochim Biophys Acta 1838: 1518-1528, 2014.
36. Prades J, Funari SS, Escribá PV and Barceló F: Effects of unsaturated fatty acids and triacylglycerols on phosphatidylethanolamine membrane structure. J Lipid Res 44: 1720-1727, 2003.

37. Appel LJ, Miller ER III, Seidler AJ and Whelton PK: Does supplementation of diet with 'fish oil' reduce blood pressure? A meta-analysis of controlled clinical trials. Arch Intern Med 153: 1429-1438, 1993.

38. Geleijnse JM, Giltay EJ, Grobbee DE, Donders AR and Kok FJ: Blood pressure response to fish oil supplementation: Metaregression analysis of randomized trials. J Hypertens 20: 1493-1499, 2002

39. Colussi G, Catena C, Novello M, Bertin N and Sechi LA: Impact of omega-3 polyunsaturated fatty acids on vascular function and blood pressure: Relevance for cardiovascular outcomes. Nutr Metab Cardiovasc Dis 27: 191-200, 2017. 